\title{
Improvements to Satisfiability-Based Boolean Function Bi-Decomposition
}

\author{
Huan Chen and Joao Marques-Silva \\ Complex \& Adaptive Systems Laboratory (CASL) \\ School of Computer Science and Informatics (CSI) \\ University College Dublin \\ Dublin, Ireland \\ $\{$ huan.chen, jpms\}@ucd.ie
}

\begin{abstract}
Boolean function bi-decomposition is pervasive in logic synthesis. Bi-decomposition entails the decomposition of a Boolean function into two other functions connected by a simple two-input gate. Existing solutions are based on Binary Decision Diagrams (BDDs) and, more recently, on Boolean Satisfiability (SAT). Recent work exploited the identification of Minimally Unsatisfiable Subformulas (MUSes) for computing the sets of variables to use in Boolean function bi-decomposition. This paper develops new techniques for improving the use of MUSes in function bi-decomposition. The first technique exploits structural properties of the function being decomposed, whereas the second technique exploits group-oriented MUSes. Experimental results obtained on representative benchmarks from logic synthesis demonstrate significant improvements both in performance and in the quality of decompositions.
\end{abstract}

Keywords: bi-decomposition, logic synthesis, satisfiability, MUS, grouporiented MUS.

\section{Introduction}

Boolean function decomposition [1, 2, is ubiquitous in logic synthesis, being a fundamental technique in multi-level logic synthesis. The goal of functional decomposition is to represent a complex Boolean function $f(X)$ as $f(X)=$ $h\left(g_{1}(X), \ldots, g_{m}(X)\right)$, such that $h, g_{1}, \ldots, g_{m}$ are simpler subfunctions. Functional decomposition plays an important role in Electronic Design Automation (EDA) for VLSI, including multi-level logic synthesis and FPGA synthesis [3 5].

Bi-decomposition [6 12 is a special form (with $m=2$ ) of Boolean function decomposition, and it is arguably the most widely used form of Boolean function decomposition. It consists of decomposing Boolean function $f(X)$ into the form of $f(X)=h\left(f_{A}\left(X_{A}, X_{C}\right), f_{B}\left(X_{B}, X_{C}\right)\right)$, under variable partition $X=$ $\left\{X_{A}\left|X_{B}\right| X_{C}\right\}$. The quality of bi-decomposition is mainly determined by the quality of variable partitions, as an optimal solution results in simpler subfunctions $f_{A}$ and $f_{B}$. Typically, two relative quality metrics [11, 13, namely disjointness and balancedness, are used to evaluate the resulting variable partitions, for which smaller values represent preferred bi-decompositions. In practice,

S. Mir et al. (Eds.): VLSI-SoC 2011, IFIP AICT 379, pp. 52-72, 2012.

(C) IFIP International Federation for Information Processing 2012 
disjointness is in general preferred [1], since it represents the reduction of common variables to $f_{A}$ and $f_{B}$, which in turn often simplifies the resulting Boolean function. Similar to recent work on functional decomposition [11,13], this paper addresses these two relative metrics, namely disjointness and balancedness. $A b$ solute quality metrics are an alternative to relative quality metrics, and include total variable count $(\Sigma)$ and maximum partition size $(\Delta)[12$. Nevertheless, in practice absolute quality metrics scale worse with the number of inputs [12].

The research on decomposition of Boolean functions can be traced back to the 1950s [1,2]. The very first algorithm for bi-decomposition was presented for the AND case in [14]. The first solution for XOR case was given in [15]. The general case of bi-decomposing of Boolean network was proposed in [16. Traditional algorithms [3, 5, 8, 10, 17] use BDDs as the underlying data structure. However, BDDs impose severe constraints on the number of input variables circuits can have. Hence, it is generally accepted that BDDs do not scale for large Boolean functions. As a result, recent work [11,13, 18, proposed the use of Boolean Satisfiability (SAT) to manipulate large Boolean functions. For example, [1] has a number of key features, including: (1) good performance on some large circuits; and (2) capability to automatically identify variable partitions. Nevertheless, detailed experimental evaluation of the work in [11 revealed a few shortcomings: (1) The ever-increasing size of circuits to synthesize requires more efficient techniques for Boolean function bi-decomposition; and (2) The underlying SAT solver affects the efficiency of computing of Minimally Unsatisfiable Subformulas (MUSes), which in turn determine the final quality of variable partitions.

The paper has two main contributions. The first one develops heuristics and adapts modern MUS algorithms, which offer significant performance improvements as well as better quality of computed bi-decompositions. The second contribution exploits the idea of constraint grouping 19] used in group-oriented (or high-level) MUSes [19 23. The use of group-oriented MUS extraction allows performance improvements that can exceed two orders of magnitude in comparison with the results of [11].

The paper is organized as follows. Section 2 provides the preliminaries. Section 3 reviews the models for Boolean function bi-decomposition. Section 4 proposes new Satisfiability-based models. Section 5 illustrates an example of OR bi-decomposition in detail. Section 6 presents the experimental results. Section 7 concludes the paper and outlines a number of future research directions.

\section{Preliminaries}

\subsection{Notation}

Variables are represented by set $X=\left\{x_{1}, x_{2}, \ldots, x_{n}\right\}$. The cardinality of $X$ is denoted as $\|X\|$. A partition of a set $X$ into $X_{i} \subseteq X$ for $i=1, \ldots, k$ (with $X_{i} \bigcap X_{j}=\emptyset, i \neq j$ and $\left.\bigcup_{i} X_{i}=X\right)$ is denoted by $\left\{X_{1}\left|X_{2}\right| \ldots \mid X_{k}\right\}$. A Completely Specified Function (CSF) is denoted by $f: \mathcal{B}^{n} \rightarrow \mathcal{B}$. An Incompletely Specified Function (ISF) $F(X)$ is a 3 -tuple $\left(f_{q}, f_{d}, f_{r}\right)$, where $f_{q}, f_{d}$ and $f_{r}$ are the onset, don't-care set and offset functions of $F(X)$. 


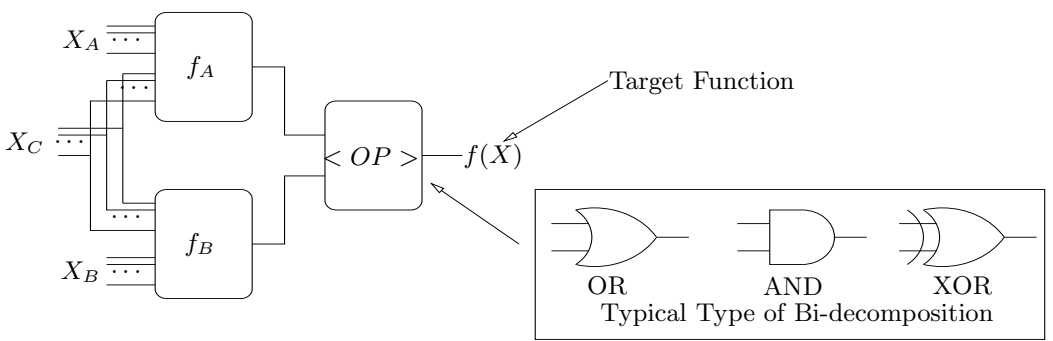

Fig. 1. Bi-decomposition

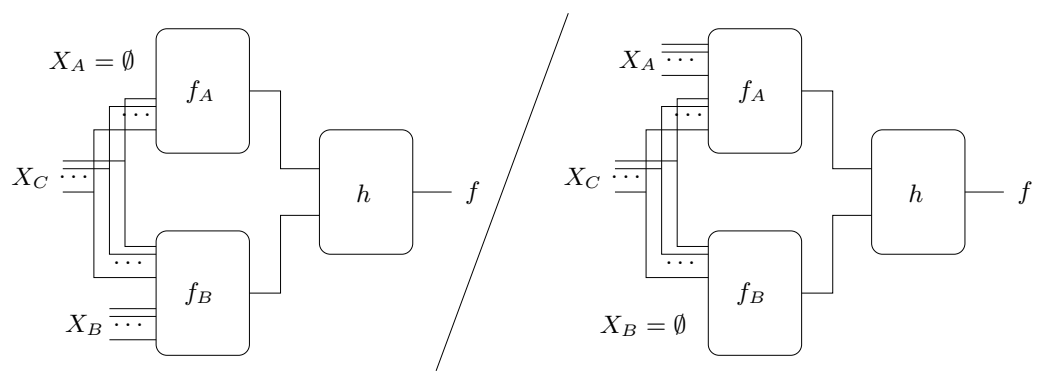

Fig. 2. Trivial variable partition

\subsection{Boolean Function Bi-Decomposition}

Definition 1 (Bi-Decomposition). [7] Bi-decomposition for a Completely Specified Function (CSF) consists of decomposing a CSF function $f(X)$ under variable partition $X=\left\{X_{A}\left|X_{B}\right| X_{C}\right\}$, into the form of $f(X)=f_{A}\left(X_{A}, X_{C}\right)$ $<O P>f_{B}\left(X_{B}, X_{C}\right)$, where $\langle O P>$ is a binary operator, typically OR, AND or $\mathrm{XOR}$.

This paper addresses $O R, A N D$ and $X O R$ bi-decomposition because these three basic gates form other types of bi-decomposition [11. Figure 1 illustrates the corresponding concepts. Bi-decomposition is termed disjoint if $\left\|X_{C}\right\|=0$. A partition of $X$ is trivial if $X=X_{A} \cup X_{C}$ or $X=X_{B} \cup X_{C}$ holds. The concept of trivial partition is illustrated in Figure 2, Similar to earlier work [11,13, this paper addresses non-trivial bi-decompositions.

Definition 2 (Support Variable). For a completely specified function $f(X)$ with input variables $X=\left(x_{1}, \ldots, x_{m}\right)$, variable $x_{i}$ is a support variable of $f$ if

$$
f\left(x_{1}, \ldots, x_{i-1}, 0, x_{i+1}, \ldots, x_{m}\right) \neq f\left(x_{1}, \ldots, x_{i-1}, 1, x_{i+1}, \ldots, x_{m}\right)
$$

\subsection{Boolean Satisfiability}

Boolean formulas are defined over a finite set $X$ of Boolean variables. Individual variables are represented by letters $x, y, z, w$ and $o$, and subscripts may be used 
(e.g. $x_{1}$ ). The Boolean connectives considered will be $\neg, \rightarrow, \leftrightarrow, \wedge, \vee$. When necessary, parentheses are used to enforce precedence.

A formula in Conjunctive Normal Form $(\mathrm{CNF}) \mathcal{F}$ is defined as a set of sets of literals defined on $X$, representing a conjunction of disjunctions of literals. A literal is either a variable or its complement. Each set of literals is referred to as a clause $c$. Moreover, it is assumed that each clause is non-tautological. The Boolean SAT problem is an NP-Complete decision problem [24. Additional SAT definitions can be found in standard references (e.g. [25]).

Definition 3 (MUS). [26] $\mathcal{M} \subseteq \mathcal{F}$ is a Minimally Unsatisfiable Subformula (MUS) iff $\mathcal{M}$ is unsatisfiable and $\forall_{c \in \mathcal{M}}, \mathcal{M} \backslash\{c\}$ is satisfiable.

MUSes find a wide range of practical applications, including Boolean function decomposition [11,13, 18, high-level MUSes [19] for the refinement of datapath abstractions [22] and formal equivalence checking [20,21]. (See [26] for a recent overview of MUSes.)

Group-oriented MUSes is an alternative name for high-level MUSes 19,21,23. In the group-oriented MUS problem, the input is an unsatisfiable set of clauses (a CNF formula) $\mathcal{C}=\mathcal{D} \cup \mathcal{G}_{1} \cup \cdots \cup \mathcal{G}_{k}$ that is explicitly partitioned into the groups $\mathcal{D}, \mathcal{G}_{1}, \ldots, \mathcal{G}_{k}$ of clauses such that $\mathcal{D} \cap \mathcal{G}_{i}=\emptyset$ and $\mathcal{G}_{i} \cap \mathcal{G}_{j}=\emptyset$ hold for each $i, j \in\{1, \ldots, k\}$ with $i \neq j$.

Definition 4 (Group-oriented MUS). [23] Given an explicitly partitioned unsatisfiable $C N F$ formula $\mathcal{C}=\mathcal{D} \cup \bigcup_{G \in \mathcal{G}} G$, where $\mathcal{G}=\left\{\mathcal{G}_{1}, \ldots, \mathcal{G}_{k}\right\}$, and $\mathcal{D}$ and each $\mathcal{G}_{i}$ are disjoint sets of clauses, a group-oriented $M U S$ of $\mathcal{C}$ is a subset $\mathcal{G}^{\prime}$ of $\mathcal{G}$ such that $\mathcal{D} \cup \bigcup_{G \in \mathcal{G}^{\prime}} G$ is unsatisfiable and, for every $\mathcal{G}^{\prime \prime} \subset \mathcal{G}^{\prime}$, we have that $\mathcal{D} \cup \bigcup_{G \in \mathcal{G}^{\prime \prime}} G$ is satisfiable.

Notice that $\mathcal{D}$ and the clauses in $\mathcal{D}$ do not contribute to the size of a grouporiented MUS, and can hence be viewed as don't care or irrelevant clauses w.r.t. the size of the group-oriented MUSes of $\mathcal{C}$. Many practical applications, e.g. 2022], require minimizing the number of high-level propositional interesting constraints in the problem formula 21. The interested constraints are expressed as sets of clauses, where those clauses can be partitioned into groups [23. For example, clauses which encode one gate of a circuit-level description may form a group. Group-oriented MUS solvers [23], e.g. MUSer [27, can identify one group-oriented MUS of $\mathcal{C}$.

\subsection{Unsatisfiability Proof and Craig Interpolation}

This subsection reviews unsatisfiability proofs and Craig Interpolation, which are used for constructing decomposition functions $f_{A}$ and $f_{B}$ in the SAT-based bi-decomposition [11. Modern SAT solvers learn clauses [28 31. For unsatisfiable instances, the original and the learned clauses can be used for generating a resolution-based unsatisfiability proof [32]. Modern SAT solvers can be instructed to generate a proof trace, which associates with each learned clause $w_{L}$, all the clauses that explain the creation of $w_{L}[32$. Given a proof trace $\Gamma$, 


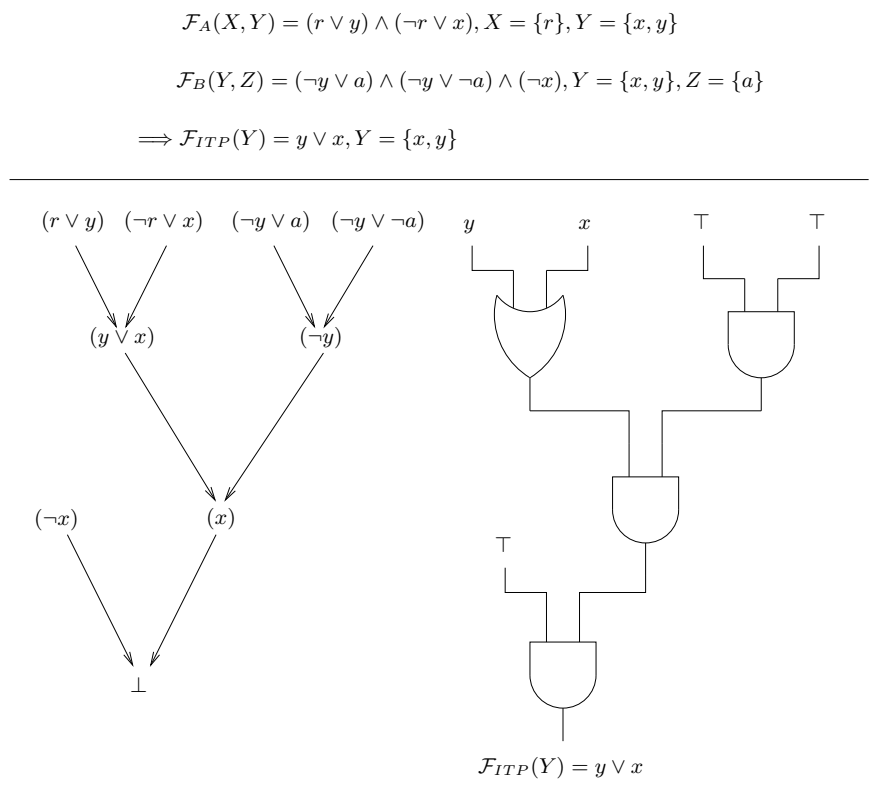

Fig. 3. Resolution graph and interpolation

where the final traced clause is an empty clause $\perp$, it is possible to create a resolution-based unsatisfiability proof in linear time and size w.r.t. proof trace.

Definition 5 (Unsatisfiability Proof e.g. [33]). A proof of unsatisfiability $\Pi$ for a set of clauses $w$ is directed acyclic graph $\left(V_{\Pi}, E_{\Pi}\right)$, where $V_{\Pi}$ is a set of clauses, such that:

- For every $w \in V_{\Pi}$, either

- $w \in \mathcal{F}$, and $w$ is a root, or

- $w$ has two predecessors, $w_{1}$ and $w_{2}$, such that $w$ is the resolvent of $w_{1}$ and $w_{2}$ (the variable $p$ used for resolving $w_{1}$ with $w_{2}$ is referred to as the pivot variable of the resolution step), and

- the empty clause $\perp$ is the unique leaf.

Assume a Boolean formula $\mathcal{F}_{A}(X, Y)$, defined over the sets of variables $X$ and $Y$, and a Boolean formula $\mathcal{F}_{B}(Y, Z)$, defined over the sets of variables $Y$ and $Z$.

Theorem 1 (Craig Interpolation). 34 If $\mathcal{F}_{A} \wedge \mathcal{F}_{B}$ is unsatisfiable, then there exists a Boolean formula $\mathcal{F}_{I T P}(Y)$, defined over the set of variables $Y$, such that $\mathcal{F}_{A}(X, Y) \rightarrow \mathcal{F}_{I T P}(Y)$ is a tautology and $\mathcal{F}_{I T P} \wedge \mathcal{F}_{B}(Y, W)$ is unsatisfiable. $\mathcal{F}_{I T P}(Y)$ is referred to as an interpolant for $\mathcal{F}_{A}(X, Y)$ and $\mathcal{F}_{B}(Y, Z)$.

Interpolant $\mathcal{F}_{I T P}$ can be computed in linear time on the size of a resolution refutation of $\mathcal{F}_{A}$ and $\mathcal{F}_{B}$ 33. Besides, $\mathcal{F}_{I T P}$ has size linear on the size of unsatisfiability proof [33, 35]. In the following, McMillan's interpolant construction [33] 
is outlined, but Pudlak's construction 35 could also be considered. Assume Boolean formulas $\mathcal{F}_{A}(X, Y)$ and $\mathcal{F}_{B}(Y, Z)$. Variables $Y$ is referred to as global variables w.r.t. $\mathcal{F}_{A}(X, Y)$ and $\mathcal{F}_{B}(Y, Z)$, whereas variables $X$ and $Z$ are local to $\mathcal{F}_{A}$ and $\mathcal{F}_{B}$ respectively. Let $g(w)$ denote the literals corresponding to global variables in clause $w$.

Definition 6 (Interpolatnt). Let $\left(\mathcal{F}_{A}, \mathcal{F}_{B}\right)$ be a pair of clause sets and let $\Pi$ be a proof of unsatisfiability of $\mathcal{F}_{A}$ and $\mathcal{F}_{B}$, with leaf vertex $\perp$. For each vertex $w \in V_{\Pi}$, let $\mathcal{F}_{w}$ be a Boolean formula, such that:

- If $w$ is root then

- if $w \in \mathcal{F}_{A}$ then $\mathcal{F}_{w}=g(w)$,

- else $\mathcal{F}_{w}=$ TRUE.

- else, let $w_{1}, w_{2}$ be the predecessors of $w$ and let $p$ be their pivot variable

- if $p$ is local to $\mathcal{F}_{A}$, then $\mathcal{F}_{w}=\mathcal{F}_{w_{1}} \vee \mathcal{F}_{w_{2}}$,

- else $\mathcal{F}_{w}=\mathcal{F}_{w_{1}} \wedge \mathcal{F}_{w_{2}}$.

- The $\Pi$-interpolation of $\left(\mathcal{F}_{A}, \mathcal{F}_{B}\right)$, denoted $\operatorname{ITP}\left(\Pi, \mathcal{F}_{A}, \mathcal{F}_{B}\right)$ is $\mathcal{F}_{\perp}$.

Example 1 (Resolution graph and interpolation). As explained in Figure 3, resolution graph of an UNSAT Boolean formula $\mathcal{F}_{A}(X, Y) \wedge \mathcal{F}_{B}(Y, Z)$ leads to an empty clause $\perp$. The interpolation procedure (see above) produces an interpolant $\mathcal{F}_{I T P}(Y)$.

\subsection{Quality Metrics}

The quality of variable partitions mainly impacts the quality of bi-decomposition [11,12, and indirectly impacts the decomposed network, e.g. delay, area and power consumption [12. Similar to [11,13, this paper measures the quality of variable partitions through two relative quality metrics, namely disjointness and balancedness. Assume a variable partition $\left\{X_{A}\left|X_{B}\right| X_{C}\right\}$ for $f(X)$, where $X_{A}, X_{B}$ and $X_{C}$ are the sets of the input variables to decomposition functions $f_{A}, f_{B}$ and common to $f_{A}$ and $f_{B}$, respectively.

Definition 7 (Disjointness). $\epsilon_{D}=\frac{\left\|X_{C}\right\|}{\|X\|}$ denotes the ratio of the number of common variables to inputs. A value of $\epsilon_{D}$ close to 0 is preferred, as $\epsilon_{D}=0$ represents a disjoint bi-decomposition.

Definition 8 (Balancedness). $\epsilon_{B}=\frac{\left|\left\|X_{A}||-|| X_{B}\right\|\right|}{\|X\|}$ denotes the absolute size difference between $X_{A}$ and $X_{B}, \epsilon_{B}=0$ represents a balanced variable partition.

In practice, disjointness is preferred since a lower value represents a smaller number of shared input variables of the resulting decomposed circuit that typically has smaller area and power footprint. A lower balancedness typically corresponds to smaller delay of the decomposed network.

\section{Related Work}

Boolean function decomposition approaches are either based on BDDs or SAT. This section briefly overviews earlier work on Boolean function decomposition. 


\subsection{BDD-Based Bi-Decomposition}

Traditional algorithms [3, 5, 8, 10, 17] of bi-decomposition are based on BDDs. BDD-based function decomposition approaches implement different forms of bidecomposition, including OR, AND, XOR, MIN and MAX 7,8, targeting optimization of timing [36] and area of the synthesized circuits 9, 10. Assuming the variable partition $X=\left\{X_{A}\left|X_{B}\right| X_{C}\right\}$ of $f(X)$ is given, then the bi-decomposition problem can be stated as follows [8]:

Definition 9. A completely specified function $f(X)$ can be written as $f_{A}\left(X_{A}, X_{C}\right) \vee f_{B}\left(X_{B}, X_{C}\right)$ for some functions $f_{A}$ and $f_{B}$ if and only if the $B D D$ quantified formula:

$$
f\left(X_{A}, X_{B}, X_{C}\right) \wedge \exists_{X_{A}} \cdot \neg f\left(X_{A}, X_{B}, X_{C}\right) \wedge \exists_{X_{B}} \cdot \neg f\left(X_{A}, X_{B}, X_{C}\right)
$$

is false.

Algorithms based on BDDs have a number of advantages, including flexible Boolean function manipulation [10, the ability to handle don't-care conditions 8 and on-demand selection of best partition of variables [37. In contrast, the main drawback of BDDs is that they can be used only on functions with a fairly small number of inputs [11].

\subsection{SAT-Based Bi-Decomposition}

Recent work 11 proposed SAT-based solutions. The use of SAT not only makes the computation of bi-decomposition feasible for large circuits, but also serves for automatically selecting and optimizing variable partitions. SAT-based OR, AND and XOR bi-decompositions under known and unknown partition of variables were proposed in [11. For example, the widely used OR bi-decomposition can be constructed by SAT solving 11. Given a non-trivial variable partition $X=$ $\left\{X_{A}\left|X_{B}\right| X_{C}\right\}$, the following result holds:

Proposition 1. [11] $A$ completely specified function $f(X)$ can be written as $f_{A}\left(X_{A}, X_{C}\right) \vee f_{B}\left(X_{B}, X_{C}\right)$ for some functions $f_{A}$ and $f_{B}$ if and only if the Boolean formula

$$
f\left(X_{A}, X_{B}, X_{C}\right) \wedge \neg f\left(X_{A}^{\prime}, X_{B}, X_{C}\right) \wedge \neg f\left(X_{A}, X_{B}^{\prime}, X_{C}\right)
$$

is unsatisfiable, where variable set $Y^{\prime}$ is an instantiated version of variable set $Y$.

An instantiated version $x^{\prime}$ of Boolean variable $x$ can be viewed as a new Boolean variable $x^{\prime}$ that replaces $x$. This approach assumes that a variable partition $X=\left\{X_{A}\left|X_{B}\right| X_{c}\right\}$ is given. In practice, such variable partitions are generally unknown and must be automatically derived. One approach to consider instead is the following formulation [11:

$$
f(X) \wedge \neg f\left(X^{\prime}\right) \wedge \bigwedge_{i}\left(\left(x_{i} \equiv x_{i}^{\prime}\right) \vee \alpha_{x_{i}}\right) \wedge \neg f\left(X^{\prime \prime}\right) \wedge \bigwedge_{i}\left(\left(x_{i} \equiv x_{i}^{\prime \prime}\right) \vee \beta_{x_{i}}\right)
$$


where $x^{\prime} \in X^{\prime}$ and $x^{\prime \prime} \in X^{\prime \prime}$ are the instantiated version of $x \in X . \alpha_{x_{i}}$ and $\beta_{x_{i}}$ are control variables for enumerating variable partitions. By assigning different Boolean values to $\alpha_{x_{i}}$ and $\beta_{x_{i}}$, some of the clauses $\left(\left(x_{i} \equiv x_{i}^{\prime}\right) \vee \alpha_{x_{i}}\right),\left(\left(x_{i} \equiv\right.\right.$ $\left.\left.x_{i}^{\prime \prime}\right) \vee \beta_{x_{i}}\right)$ are relaxed. The resulting clauses $\left(x_{i} \equiv x_{i}^{\prime}\right)$ and $\left(x_{i} \equiv x_{i}^{\prime \prime}\right)$ impose equivalence relations for each pair of variables in sets $X$ and $X^{\prime}$, and in $X$ and $X^{\prime \prime}$, respectively.

The original work on SAT-based bi-decomposition [11] proposed the use of interpolation for computing the target functions $f_{A}$ and $f_{B}$. Given that our work focuses on improving the identification of MUSes, interpolation can also be used for computing functions $f_{A}$ and $f_{B}$. Similar to OR bi-decomposition, AND and XOR bi-decomposition can be constructed by using Boolean SAT. Due to space limitations, this section omits the explanation of SAT-based AND and XOR bi-decompositions (e.g. see [1]). The approaches proposed [1] are referred to as $L J H$ in the remainder of the paper.

\section{Improved MUS-Based Bi-Decomposition}

Earlier work on SAT-based function bi-decomposition proposed computing MUSes with SAT solvers [11,13, 18, where partitions are partially enumerated. This section extends this earlier work, and develops two techniques that improve performance significantly and achieve better quality partitions. The first technique exploits structural properties for guiding the computation of MUSes. The second technique exploits recent work on applying group-oriented MUSes in formal verification of large-scale designs 1922 .

\subsection{Plain MUS-Based Bi-Decomposition}

OR Bi-Decomposition for CSF. OR bi-decomposition can be constructed by SAT solving [11]. Given a non-trivial variable partition $X=\left\{X_{A}\left|X_{B}\right| X_{C}\right\}$, a CSF $f(X)$ can be written as $f_{A}\left(X_{A}, X_{C}\right) \vee f_{B}\left(X_{B}, X_{C}\right)$ for some functions $f_{A}$ and $f_{B}$ iff the Boolean formula

$$
f\left(X_{A}, X_{B}, X_{C}\right) \wedge \neg\left(X_{A}^{\prime}, X_{B}, X_{C}\right) \wedge \neg f\left(X_{A}, X_{B}^{\prime}, X_{C}\right)
$$

is unsatisfiable. This approach assumes that a variable partition $X=\left\{X_{A}\left|X_{B}\right| X_{C}\right\}$ is given. In practice, such variable partitions are generally unknown and need to be automatically derived. As a result, the derivation of variable partitions must be automated. Earlier work [11] proposed the SAT-based model given in (4). This model gives a variable partition if (4) is unsatisfiable under a non-trivial partition. $\alpha_{x_{i}}$ and $\beta_{x_{i}}$ are called control variables, used for the purpose of relaxing clauses. Assignments $\left(\alpha_{x_{i}}, \beta_{x_{i}}\right)=(0,0),(0,1),(1,0)$ and $(1,1)$ indicate the partition, to which $x_{i}$ belongs, $x_{i} \in X_{C}, x_{i} \in X_{B}, x_{i} \in X_{A}$, and $x_{i}$ can either be in $X_{A}$ or $X_{B}$, respectively.

Enumerating different values of the control variables will result in different variable partitions. A solution corresponds to an Unsatisfiable Subformula (US) of the original CNF formula. An optimal solution is an MUS. The optimization 
of variable partitions is the process of enumerating and selecting MUSes. If a disjoint variable partition $\left(\left\|X_{C}\right\|=0\right)$ is concerned, the solving process corresponds to finding a minimum unsatisfiable core 11. However, it is well-known that computing a minimum-size unsatisfiable core is harder than computing a minimal one. Therefore, a practical solution is to compute an MUS instead.

Equation (4) serves to extract an unsatisfiable subformula that results in a non-trivial partition. This is done by enumerating control variables. However, this enumeration is known not to be effective in practice, essentially because the enumeration is exponential in the number of variables. As a result, this SAT-based model is modified such that (1) control variables are removed, (2) structural heuristics are used to guide the search for a partition, and (3) the interface of a modern MUS extractor MUSer [27] is exploited to improve overall performance.

Proposition 2. A completely specified function $f(X)$ can be decomposed into $f_{A}\left(X_{A}, X_{C}\right) \vee f_{B}\left(X_{B}, X_{C}\right)$ for some functions $f_{A}$ and $f_{B}$ if and only if the Boolean formula

$$
f(X) \wedge \neg f\left(X^{\prime}\right) \wedge \mathcal{F}_{\mathcal{A}} \wedge \neg f\left(X^{\prime \prime}\right) \wedge \mathcal{F}_{\mathcal{B}}
$$

is unsatisfiable under a non-trivial partition, where $\mathcal{F}_{\mathcal{A}} \subset \bigcup_{i}\left\{\left(x_{i} \equiv x_{i}^{\prime}\right)\right\}$, and $\mathcal{F}_{\mathcal{B}} \subset \bigcup_{i}\left\{\left(x_{i} \equiv x_{i}^{\prime \prime}\right)\right\}$, variable set $X^{\prime}$ and $X^{\prime \prime}$ are the instantiated versions of variable set $X, x^{\prime} \in X^{\prime}$ and $x^{\prime \prime} \in X^{\prime \prime}$ are the instantiated versions of $x \in X$.

The identification of a non-trivial variable partition typically starts from identifying a seed variable partition [11. A seed variable partition makes (4) unsatisfiable where partition $X_{A}$ and $X_{B}$ each take at least one variable. This scheme also applies to the proposed new model (6). It can be shown that the existence of non-trivial OR bi-decomposition can be checked with at most $C_{n}^{2}=(n-1)+\cdots+1=\frac{n(n-1)}{2}$ different seed partitions [11. Figure 4 shows a AIG (And-Inverter Graph), representing a disjointly decomposable circuit. A normal search may first check $X_{A}=\{c\}, X_{B}=\{d\}$ and $X_{C}=\{a, b\}$. Unfortunately, this results in a trivial partition. Afterwards, since the SAT check failed,

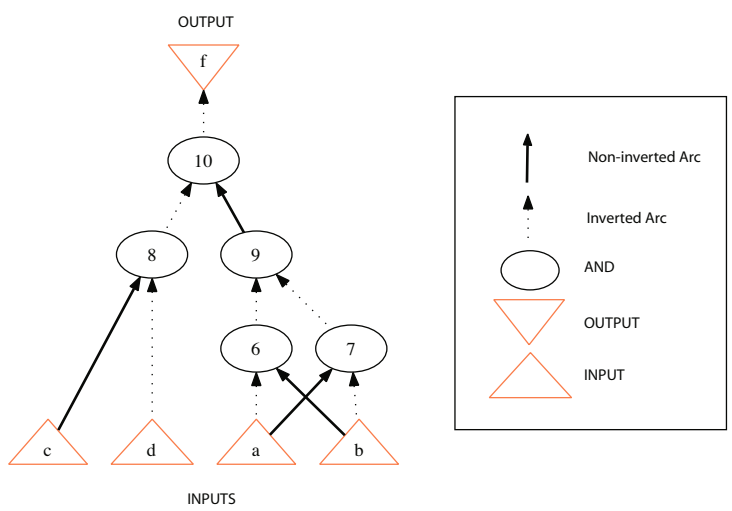

Fig. 4. AIG with disjoint variable partition 
the algorithm enumerates other combinations of inputs for $X_{A}$ and $X_{B}$ until gets a non-trivial partition. Heuristically, the search of seed variables can incorporate circuit structural information. Selecting one non-common input variable $l(l \in$ leaf of left-subtree while $l \notin$ leaf of right-subtree, or $l \notin$ leaf of left-subtree while $l \in$ leaf of right-subtree, if possible) from leaves in each subtree of the root node raises the likelihood of getting seed variables in part because the AIGs are structurally hashed. For example in Figure 4, simply select $X_{A}=\{c\}$ from the left subtree and $X_{B}=\{a\}$ from the right subtree shapes a seed partition. In practice, this heuristic will help to quickly form the seed variable partitions.

XOR Bi-Decomposition for CSF. Similar to the proposed modification to OR bi-decomposition, the XOR bi-decomposition can be constructed by a succinct form of MUS-based model through removing the control variables.

Proposition 3. A completely specified function $f(X)$ can be decomposed into $f_{A}\left(X_{A}, X_{C}\right) \oplus f_{B}\left(X_{B}, X_{C}\right)$ for some functions $f_{A}$ and $f_{B}$ if and only if the Boolean formula

$$
\left(f(X) \equiv f\left(X^{\prime}\right)\right) \wedge\left(f\left(X^{\prime \prime}\right) \not \equiv f\left(X^{\prime \prime \prime}\right)\right) \wedge \mathcal{F}_{\mathcal{A}} \wedge \mathcal{F}_{\mathcal{B}}
$$

is unsatisfiable under a non-trivial partition, where the sub-formula $\mathcal{F}_{\mathcal{A}} \subset$ $\bigcup_{i}\left\{\left(\left(x_{i} \equiv x_{i}^{\prime \prime}\right) \wedge\left(x_{i}^{\prime} \equiv x_{i}^{\prime \prime \prime}\right)\right)\right\}$, the sub-formula $\mathcal{F}_{\mathcal{B}} \subset \bigcup_{i}\left\{\left(\left(x_{i} \equiv x_{i}^{\prime}\right) \wedge\left(x_{i}^{\prime \prime} \equiv x_{i}^{\prime \prime \prime}\right)\right)\right\}$, variable set $X^{\prime}, X^{\prime \prime}$ and $X^{\prime \prime \prime}$ are the instantiated versions of variable set $X$, $x^{\prime} \in X^{\prime}, x^{\prime \prime} \in X^{\prime \prime}$ and $x^{\prime \prime \prime} \in X^{\prime \prime \prime}$ are the instantiated versions of $x \in X$.

\subsection{Group-Oriented MUS-Based Bi-Decomposition}

OR Bi-Decomposition for CSF. Essentially, the derivation of variable partitions is the process of switching the input variables between the two partitions. Interestingly, this switching behaviour can be captured by selecting the groups of the input variables. Partition the clauses of formula (6) into $(2 i+1)$ groups:

$$
\begin{array}{r}
\mathcal{D}=\left\{f(X) \wedge \neg f\left(X^{\prime}\right) \wedge \neg f\left(X^{\prime \prime}\right)\right\} \\
\mathcal{G}_{i_{a}}=\left\{\left(x_{i} \equiv x_{i}^{\prime}\right)\right\} \\
\mathcal{G}_{i_{b}}=\left\{\left(x_{i} \equiv x_{i}^{\prime \prime}\right)\right\}
\end{array}
$$

Proposition 4. A completely specified function $f(X)$ can be decomposed into $f_{A}\left(X_{A}, X_{C}\right) \vee f_{B}\left(X_{B}, X_{C}\right)$ for some functions $f_{A}$ and $f_{B}$ if and only if the Boolean formula of the set of clauses $\mathcal{C}$, with

$$
\mathcal{C}=\mathcal{D} \cup \mathcal{G}_{\mathcal{A}} \cup \mathcal{G}_{\mathcal{B}}
$$

is unsatisfiable under a non-trivial partition, where the sub-set $\mathcal{G}_{\mathcal{A}} \subset\left\{\bigcup_{i} \mathcal{G}_{i_{a}}\right\}$, the sub-set $\mathcal{G}_{\mathcal{B}} \subset\left\{\bigcup_{i} \mathcal{G}_{i_{b}}\right\}$.

Observe that the resulting subset $\mathcal{C}^{\prime}=\mathcal{D} \cup \bigcup_{i} \mathcal{G}_{i_{a}}^{\prime} \cup \bigcup_{i} \mathcal{G}_{i_{b}}^{\prime}$ from solving (99) indicates the variable partitions, where $\mathcal{G}_{i_{a}}^{\prime}$ and $\mathcal{G}_{i_{b}}^{\prime}$ with $\left(\left(\mathcal{G}_{i_{a}}^{\prime} \equiv \mathcal{G}_{i_{a}}\right),\left(\mathcal{G}_{i_{b}}^{\prime} \equiv \mathcal{G}_{i_{b}}\right)\right)=$ $(1,1),(1,0),(0,1)$, and $(0,0)$ indicate $x_{i} \in X_{C}, x_{i} \in X_{B}, x_{i} \in X_{A}$ and $x_{i}$ can 
be in either of $X_{A}$ and $X_{B}$, respectively. $\mathcal{D}$ consists of $f(X), f\left(X^{\prime}\right)$ and $f\left(X^{\prime \prime}\right)$, which is considered as the don't-care group. Clauses in this group are irrelevant for MUS extraction; this explains in part the performance improvements observed. As stated earlier, group-oriented MUS extraction must operate on an unsatisfiable formula. Similar to the plain MUS-based approach, a computed seed partition serves as an initial unsatisfied formula of (9).

XOR Bi-Decomposition for CSF. The XOR bi-decomposition for CSF can be constructed in a similar way to the group-oriented MUS-based OR bidecomposition. Partition the clauses of formula (7) into $(2 i+1)$ groups:

$$
\begin{array}{r}
\mathcal{D}=\left\{\left(f(X) \equiv f\left(X^{\prime}\right)\right) \wedge\left(f\left(X^{\prime \prime}\right) \not \equiv f\left(X^{\prime \prime \prime}\right)\right)\right\} \\
\mathcal{G}_{i_{a}}=\left\{\left(\left(x_{i} \equiv x_{i}^{\prime \prime}\right) \wedge\left(x_{i}^{\prime} \equiv x_{i}^{\prime \prime \prime}\right)\right)\right\} \\
\mathcal{G}_{i_{b}}=\left\{\left(\left(x_{i} \equiv x_{i}^{\prime}\right) \wedge\left(x_{i}^{\prime \prime} \equiv x_{i}^{\prime \prime \prime}\right)\right)\right\}
\end{array}
$$

Proposition 5. A completely specified function $f(X)$ can be decomposed into $f_{A}\left(X_{A}, X_{C}\right) \oplus f_{B}\left(X_{B}, X_{C}\right)$ for some functions $f_{A}$ and $f_{B}$ if and only if the Boolean formula of the set of clauses $\mathcal{C}$, with

$$
C=\mathcal{D} \cup \mathcal{G}_{\mathcal{A}} \cup \mathcal{G}_{\mathcal{B}}
$$

is unsatisfiable under a non-trivial partition, where the sub-set $\mathcal{G}_{\mathcal{A}} \subset\left\{\bigcup_{i} \mathcal{G}_{i_{a}}\right\}$, the sub-set $\mathcal{G}_{\mathcal{B}} \subset\left\{\bigcup_{i} \mathcal{G}_{i_{b}}\right\}$.

\subsection{AND Bi-Decomposition}

AND bi-decomposition is dual to OR bi-decomposition and can be obtained from the construction of OR bi-decomposition [8, 10, 11]. The proposed MUS model (6) is able to decompose $\neg f$ into $f_{A} \vee f_{B}$. By negating both sides, $f$ is decomposed into $\neg f_{A} \wedge \neg f_{B}$ [11. Because AIGs (And-Inverter Graphs) are used for manipulating the circuit network, the above conversion could be performed by rewriting only part of the AIG network used in OR bi-decomposition. The following proposition is used to assert the existence and correctness of AND bi-decomposition from the construction of OR bi-decomposition.

Proposition 6. [11] A function $f$ is AND bi-decomposable if and only if $\neg f$ is OR bi-decomposable.

\subsection{Incompletely Specified Function (ISF)}

This paper assumes Completely Specified Function (CSF). Incompletely Specified Function (ISF) $F(X)=\left(f_{q}, f_{d}, f_{r}\right)$ can be decomposed by searching a completely specified function $f$ with $f(X)=f_{A}\left(X_{A}, X_{C}\right) \vee f_{B}\left(X_{B}, X_{C}\right), f_{q}(X) \Rightarrow$ $f(X), f(X) \Rightarrow \neg f_{r}(X)$ if and only if

$$
f_{q}\left(X_{A}, X_{B}, X_{C}\right) \wedge f_{r}\left(X_{A}^{\prime}, X_{B}, X_{C}\right) \wedge f_{r}\left(X_{A}, X_{B}^{\prime}, X_{C}\right)
$$

is unsatisfiable [10,11]. 


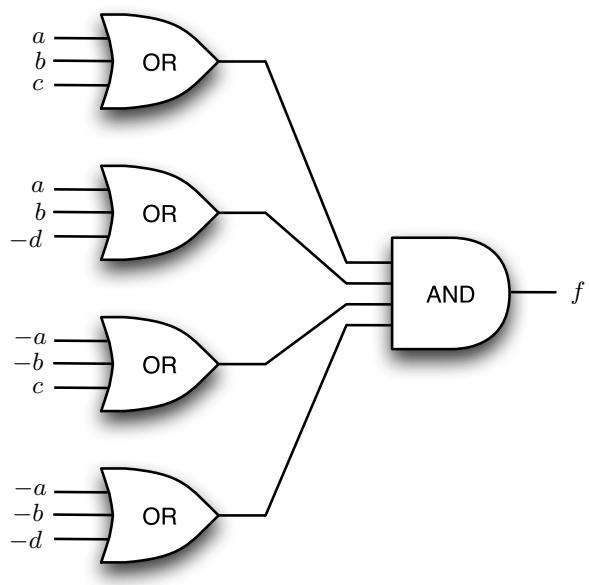

Fig. 5. Circuit network of function $f(a, b, c, d)$

\section{An Example of OR Bi-Decomposition}

This section gives an example of plain-MUS-based OR bi-decomposition for a better understanding of the techniques proposed in this paper.

Example 2 (Plain-MUS based OR Bi-decomposition). To OR bi-decompose a Boolean function $f(a, b, c, d)$, shown as Figure 5 in the Product-of-Sums (PoS) form:

$$
f(a, b, c, d)=(a \vee b \vee c) \wedge(a \vee b \vee \neg d) \wedge(\neg a \vee \neg b \vee c) \wedge(\neg a \vee \neg b \vee \neg d)
$$

where the inputs are $X=\{a, b, c, d\}$. Assume the variable partition is unknown.

Bi-decomposing of a Boolean function starts from encoding the constraints into CNF. Threefold Boolean functions are required to be encoded:

- $f(X)$ : the original Boolean function;

- $f\left(X^{\prime}\right)$ : the instantiated version of $f(X)$, where the variables in $f(X)$ are all replaced by fresh new variables;

- $f\left(X^{\prime \prime}\right)$ : the instantiated version of $f(X)$, where the variables in $f(X)$ are all replaced by fresh new variables.

In addition, clauses for encoding the equivalent relationship between the input variables $X$ are required:

$$
\begin{aligned}
& \left(a \vee \neg a^{\prime}\right) \wedge\left(\neg a \vee a^{\prime}\right) \wedge\left(a \vee \neg a^{\prime \prime}\right) \wedge\left(\neg a \vee a^{\prime \prime}\right) \\
& \wedge\left(b \vee \neg b^{\prime}\right) \wedge\left(\neg b \vee b^{\prime}\right) \wedge\left(b \vee \neg b^{\prime \prime}\right) \wedge\left(\neg b \vee b^{\prime \prime}\right) \\
& \wedge\left(c \vee \neg c^{\prime}\right) \wedge\left(\neg c \vee c^{\prime}\right) \wedge\left(c \vee \neg c^{\prime \prime}\right) \wedge\left(\neg c \vee c^{\prime \prime}\right) \\
\wedge & \left(d \vee \neg d^{\prime}\right) \wedge\left(\neg d \vee d^{\prime}\right) \wedge\left(d \vee \neg d^{\prime \prime}\right) \wedge\left(\neg d \vee d^{\prime \prime}\right)
\end{aligned}
$$




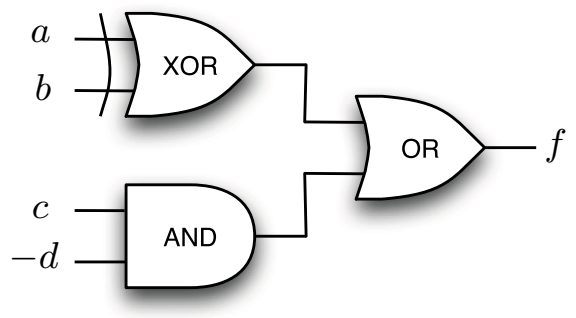

Fig. 6. Bi-decomposed network of function $f(a, b, c, d)$

After the encoding of constraints, the next step is to find a seed variable partition with the proposed techniques shown as Figure 4. As a result, an UNSAT formula with non-trivial variable partition is formed:

$$
\begin{aligned}
& f(X) \wedge \neg f\left(X^{\prime}\right) \wedge \neg f\left(X^{\prime \prime}\right) \\
& \wedge\left(a \vee \neg a^{\prime \prime}\right) \wedge\left(\neg a \vee a^{\prime \prime}\right) \\
& \wedge\left(b \vee \neg b^{\prime}\right) \wedge\left(\neg b \vee b^{\prime}\right) \wedge\left(b \vee \neg b^{\prime \prime}\right) \wedge\left(\neg b \vee b^{\prime \prime}\right) \\
& \wedge\left(c \vee \neg c^{\prime}\right) \wedge\left(\neg c \vee c^{\prime}\right) \\
& \wedge\left(d \vee \neg d^{\prime}\right) \wedge\left(\neg d \vee d^{\prime}\right) \wedge\left(d \vee \neg d^{\prime \prime}\right) \wedge\left(\neg d \vee d^{\prime \prime}\right)
\end{aligned}
$$

where $a \in X_{A}, c \in X_{B}, b \in X_{C}, d \in X_{C}$.

The MUS search of (15) helps to refine the variable partitions. In this example, an MUS:

$$
\begin{array}{r}
f(X) \wedge \neg f\left(X^{\prime}\right) \wedge \neg f\left(X^{\prime \prime}\right) \\
\wedge\left(a \vee \neg a^{\prime \prime}\right) \wedge\left(\neg a \vee a^{\prime \prime}\right) \wedge\left(b \vee \neg b^{\prime \prime}\right) \wedge\left(\neg b \vee b^{\prime \prime}\right) \\
\wedge\left(c \vee \neg c^{\prime}\right) \wedge\left(\neg c \vee c^{\prime}\right) \wedge\left(d \vee \neg d^{\prime}\right) \wedge\left(\neg d \vee d^{\prime}\right)
\end{array}
$$

where $a \in X_{A}, b, \in X_{A}, c \in X_{B}, d \in X_{B}$ reflects an ideal disjoint and balanced variable partition, where disjointness $=0$ and balancedness $=0$. The search of variable partitions requires most of the CPU time in bi-decomposition [12]. In contrast, the computation of decomposition functions $f_{A}$ and $f_{B}$ is not timeconsuming in SAT-based bi-decompositions.

The Craig Interpolation serves to derive $f_{A}$ and $f_{B}$ in OR bi-decomposition [1]. Formula (5) is suggested to replace the use of formula (6).

$$
f(a, b, c, d) \wedge \neg f_{B}(c, d) \wedge \neg f_{A}(a, b)
$$

The remaining work of deriving $f_{A}$ and $f_{B}$ follows the procedure proposed [1]. Finally, the interpolation will find the decomposition functions:

$$
\begin{array}{r}
f_{A}(a, b)=a \oplus b \\
f_{B}(c, d)=c \wedge \neg d
\end{array}
$$

The corresponding bi-decomposed circuit network is shown in Figure 6 . 
Table 1. OR Bi-decomposition of Primary Output Functions

\begin{tabular}{|c|c|c|c|c|c|c|c|c|c|c|c|c|}
\hline \multirow{2}{*}{ Circuit } & \multicolumn{3}{|c|}{ Circuit Statistics } & \multicolumn{2}{|c|}{ LJH [1] } & \multicolumn{2}{|c|}{ Plain-MUS } & \multicolumn{2}{|c|}{ Group-MUS } & \multicolumn{3}{|c|}{ CPU Time Ratio } \\
\hline & \#In & \#In_Max & \#Out & \#Dec & Time $(\mathrm{s})$ & \#Dec & Time $(\mathrm{s})$ & \#Dec & Time $(\mathrm{s})$ & $\frac{L J H}{\text { Plain-MUS }}$ & $\frac{L J H}{\mid \text { Group }-M U S}$ & $\frac{\text { Plain-MUS }}{\text { Group }-M U S}$ \\
\hline s13207 & 700 & 212 & 790 & 265 & 171.27 & 264 & 57.38 & 265 & 3.21 & 2.98 & 53.36 & 17.88 \\
\hline $\mathrm{i} 2$ & 201 & 201 & 1 & 1 & 0.85 & 1 & 0.71 & 1 & 0.20 & 1.20 & 4.25 & 3.55 \\
\hline c7552 & 207 & 194 & 108 & - & TO & 16 & 97.60 & 17 & 14.03 & 6.15 & 42.77 & 6.96 \\
\hline $\mathrm{s} 15850$ & 611 & 183 & 684 & - & $\mathrm{TO}$ & 287 & 429.31 & 294 & 21.53 & 1.40 & 27.87 & 19.94 \\
\hline s38584 & 1464 & 147 & 1730 & - & $\mathrm{TO}$ & 1057 & 62.91 & 1057 & 16.67 & 9.54 & 35.99 & 3.77 \\
\hline o64 & 130 & 130 & 1 & 1 & 0.23 & 1 & 0.19 & 1 & 0.11 & 1.21 & 2.09 & 1.73 \\
\hline c2670 & 233 & 119 & 140 & 40 & 25.57 & 40 & 20.29 & 40 & 2.25 & 1.26 & 11.36 & 9.02 \\
\hline i10 & 257 & 108 & 224 & - & TO & 149 & 183.91 & 150 & 16.83 & 3.26 & 35.65 & 10.93 \\
\hline s3330 & 173 & 87 & 205 & 59 & 8.50 & 74 & 2.28 & 74 & 0.80 & 3.73 & 10.63 & 2.85 \\
\hline s9234 & 247 & 83 & 250 & 102 & 159.50 & 111 & 20.32 & 107 & 11.36 & 7.85 & 14.04 & 1.79 \\
\hline dalu & 75 & 75 & 16 & - & TO & 15 & 20.57 & 16 & 3.25 & 29.17 & 184.62 & 6.33 \\
\hline c5315 & 178 & 67 & 123 & - & TO & 79 & 41.71 & 80 & 12.94 & 14.39 & 46.37 & 3.22 \\
\hline $\mathrm{s} 838$ & 66 & 66 & 33 & 1 & 4.37 & 1 & 2.59 & 1 & 2.47 & 1.69 & 1.77 & 1.05 \\
\hline s938 & 66 & 66 & 33 & 1 & 2.47 & 1 & 2.28 & 1 & 1.79 & 1.08 & 1.38 & 1.27 \\
\hline rot & 135 & 63 & 107 & 49 & 47.22 & 62 & 2.87 & 61 & 1.29 & 16.45 & 36.60 & 2.22 \\
\hline s5378 & 214 & 61 & 228 & 108 & 39.54 & 112 & 5.67 & 112 & 1.46 & 6.97 & 27.08 & 3.88 \\
\hline s1423 & 91 & 59 & 79 & 26 & 72.65 & 41 & 7.85 & 34 & 1.47 & 9.25 & 49.42 & 5.34 \\
\hline pair & 173 & 53 & 137 & 117 & 28.49 & 114 & 8.69 & 114 & 6.11 & 3.28 & 4.66 & 1.42 \\
\hline c3540 & 50 & 50 & 22 & & TO & 10 & 142.33 & 13 & 25.75 & 4.22 & 23.30 & 5.53 \\
\hline
\end{tabular}

\section{Experimental Results}

The new techniques described in the previous sections have been implemented in the tool $\boldsymbol{S T E P}-\boldsymbol{S}$ atisfiability-based funcTion $d \boldsymbol{E}$ comPosition for Boolean function bi-decomposition. $\boldsymbol{S T E P}$ is implemented in $\mathrm{C}++$, compiled with $\mathrm{G}++$ 4.4.3, and uses $\boldsymbol{A B C} 38$ for circuit manipulation. In addition, $\boldsymbol{S T E P}$ uses MUSer 27] as the underlying MUS extractor. The tool $\boldsymbol{B} \boldsymbol{i}$-dec implements OR bi-decomposition of LJH model 11 11.

The experiments compare the performance and quality of Boolean function bidecompositions between $\boldsymbol{B} \boldsymbol{i}$ - $\boldsymbol{d e c}$ (with its fastest mode, using command ' $b i_{-} d e c$ [circuit.blif] or $00^{\prime}$ ) and $\boldsymbol{S T E P}$. All results were obtained on the industrial benchmark circuits ISCAS85, ISCAS89, ITC99 and LGSYNTH. Circuits with zero decomposable Primary Output (PO) functions were removed from the tables of results. Due to space restrictions, only representative experimental results (with \#In_Max $\geq 50$ ) are shown.

The experiments were performed on a Linux machine with an Intel CPU Xeon X3470 2.93 GHz and 6-GB RAM. The original circuits were used. Sequential circuits were converted into combinational circuits using $\boldsymbol{A} \boldsymbol{B} \boldsymbol{C}$ [38. Similar to [1],

${ }^{1}$ AND and XOR bi-decompositions using LJH model is unavailable in the tool $\boldsymbol{B i}$-dec. 
Table 2. AND and XOR Bi-decomposition of Primary Output Functions

\begin{tabular}{|c|c|c|c|c|c|c|c|c|c|c|c|c|c|}
\hline \multirow{3}{*}{ Circuit } & \multirow{2}{*}{\multicolumn{3}{|c|}{ Circuit Statistics }} & \multicolumn{4}{|c|}{ AND Bi-decomposition } & \multicolumn{4}{|c|}{ XOR Bi-decomposition } & \multicolumn{2}{|c|}{ CPU Time Ratio } \\
\hline & & & & \multicolumn{2}{|c|}{ Plain-MUS } & \multicolumn{2}{|c|}{ Group-MUS } & \multicolumn{2}{|c|}{ Plain-MUS } & \multicolumn{2}{|c|}{ Group-MUS } & \multirow{2}{*}{\begin{tabular}{|c|} 
AND \\
$\frac{\text { Plain-MUS }}{\text { Group }-M U S}$ \\
\end{tabular}} & \multirow{2}{*}{\begin{tabular}{|c|} 
XOR \\
$\frac{\text { Plain-MUS }}{\text { Group }-M U S}$
\end{tabular}} \\
\hline & $\#$ In & \#In_Max & \#Out & \#Dec & Time (s) & \#Dec & Time (s) & \#Dec & Time (s) & \#Dec & Time (s) & & \\
\hline s13207 & 700 & 212 & 790 & 299 & 43.57 & 301 & 3.58 & 260 & 63.52 & 262 & 6.69 & 12.17 & 9.49 \\
\hline $\mathrm{i} 2$ & 201 & 201 & 1 & 1 & 1.17 & 1 & 0.31 & 1 & 2.35 & 1 & 0.88 & 3.77 & 2.67 \\
\hline c7552 & 207 & 194 & 108 & 10 & 104.21 & 11 & 26.15 & 8 & 457.04 & 10 & 52.97 & 3.99 & 8.63 \\
\hline s15850 & 611 & 183 & 684 & 358 & 417.65 & 351 & 19.39 & - & TO & 237 & 63.35 & 21.54 & 9.47 \\
\hline s38584 & 1464 & 147 & 1730 & 1099 & 68.27 & 1103 & 22.83 & 963 & 173.05 & 965 & 34.55 & 2.99 & 5.01 \\
\hline o64 & 130 & 130 & 1 & 1 & 0.80 & 1 & 0.38 & 0 & 38.33 & 0 & 31.95 & 2.11 & 1.20 \\
\hline c2670 & 233 & 119 & 140 & 37 & 16.63 & 37 & 2.74 & 33 & 52.20 & 35 & 8.61 & 6.07 & 6.06 \\
\hline $\mathrm{i} 10$ & 257 & 108 & 224 & 162 & 223.99 & 171 & 19.77 & -1 & TO & 144 & 56.50 & 11.33 & 10.62 \\
\hline s3330 & 173 & 87 & 205 & 83 & 2.28 & 85 & 0.35 & 51 & 4.74 & 55 & 1.91 & 6.51 & 2.48 \\
\hline s9234 & 247 & 83 & 250 & 132 & 21.68 & 131 & 10.85 & 106 & 45.57 & 104 & 9.44 & 2.00 & 4.83 \\
\hline dalu & 75 & 75 & 16 & 16 & 19.41 & 16 & 2.21 & 15 & 40.50 & 15 & 3.29 & 8.78 & 12.31 \\
\hline c5315 & 178 & 67 & 123 & 78 & 16.62 & 78 & 3.28 & 82 & 59.80 & 82 & 10.37 & 5.07 & 5.77 \\
\hline s838 & 66 & 66 & 33 & 1 & 2.90 & 1 & 2.39 & 32 & 2.66 & 32 & 0.73 & 1.21 & 3.64 \\
\hline s938 & 66 & 66 & 33 & 1 & 2.28 & 1 & 1.76 & 32 & 1.86 & 32 & 0.63 & 1.30 & 2.95 \\
\hline rot & 135 & 63 & 107 & 71 & 2.59 & 69 & 0.69 & 22 & 14.62 & 22 & 2.61 & 3.75 & 5.60 \\
\hline s5378 & 214 & 61 & 228 & 124 & 6.02 & 124 & 1.33 & 98 & 27.26 & 98 & 9.94 & 4.53 & 2.74 \\
\hline s1423 & 91 & 59 & 79 & 53 & 10.13 & 47 & 1.00 & 64 & 15.50 & 64 & 2.69 & 10.13 & 5.76 \\
\hline pair & 173 & 53 & 137 & 121 & 9.87 & 121 & 4.94 & 98 & 24.49 & 98 & 8.75 & 2.00 & 2.80 \\
\hline c3540 & 50 & 50 & 22 & 12 & 127.07 & 14 & 41.96 & 6 & 134.61 & 9 & 44.18 & 3.03 & 3.05 \\
\hline
\end{tabular}

for comparison purposes, only experimental results of completely specified functions are shown. For each circuit, the timeout was set to 600 seconds. Each run of the MUS extraction was given a timeout of 10 seconds, that suffices even for the larger circuits.

\subsection{Performance of New Techniques}

Overall efficiency is crucial in function decomposition as logic synthesis of a circuit involves several iterations of function decomposition. This section evaluates the performance improvements of the techniques proposed in this paper. Two metrics, CPU time and the number of decomposable functions, were used for assessing performance. Smaller CPU times indicate that decomposing a complete circuit will be faster. A larger number of decomposable functions represents an enhanced decomposability of the tool, indicating the tool is able to decompose more functions in the allowed CPU time, assuming more decomposable functions do exist. Due to space restrictions, only results for circuits with large number of support sizes $(\geq 50)$ are presented. Table 1 shows the CPU times and the number of decomposable functions for OR bi-decompositions. Columns \#In, \#In_Max, \#Out, \#Dec and Time (s) denote the number of primary inputs, maximum 
OR : LJH vs. Plain-MUS

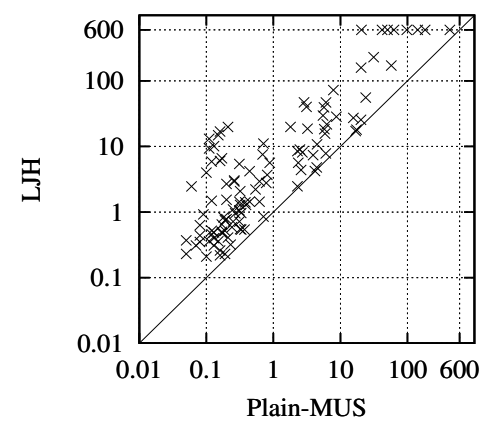

OR : LJH vs. Group-MUS

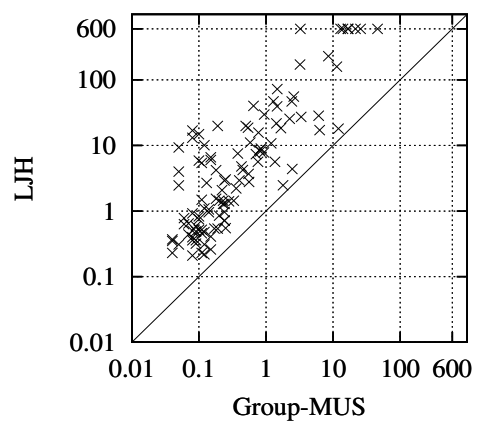

OR : Plain-MUS vs. Group-MUS

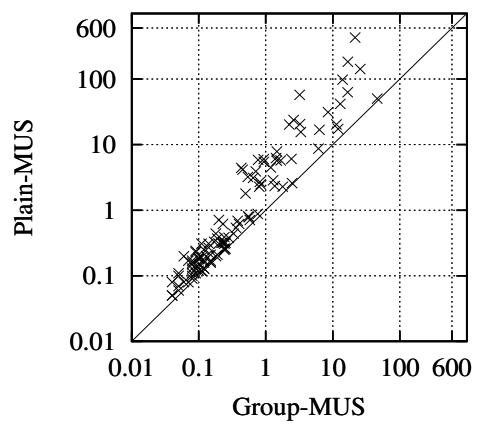

Fig. 7. CPU time comparison between models for OR bi-decomposition

AND : Plain-MUS vs. Group-MUS

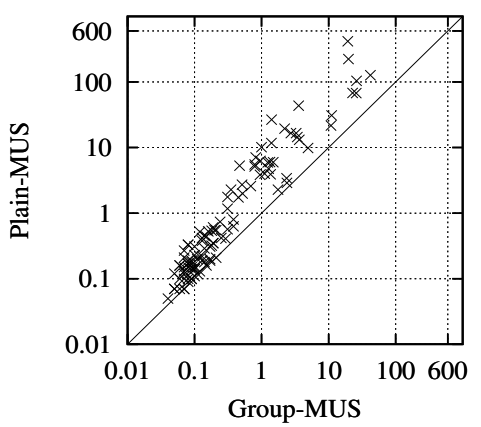

XOR : Plain-MUS vs. Group-MUS

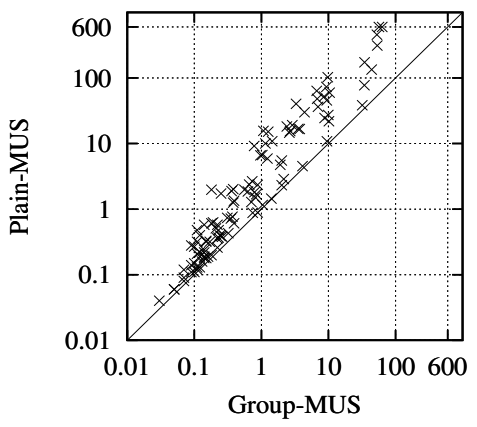

Fig. 8. CPU time comparison between models for AND/XOR bi-decomposition

number of support variables in POs, PO functions (to be decomposed) and decomposable POs and CPU time in seconds, respectively. The experimental data is sorted by decreasing number of maximum support variables (\#In_Max), to highlight the ability of $\boldsymbol{S T E P}$ at coping with large Boolean functions. The results clearly demonstrate that the techniques proposed in this paper significantly 


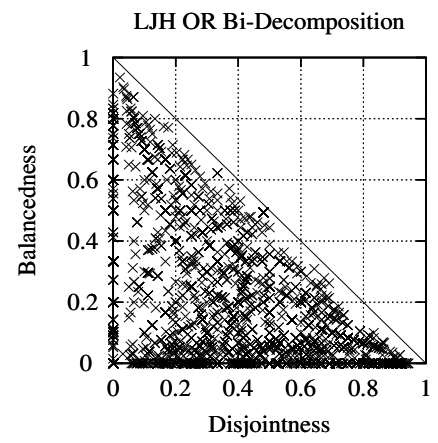

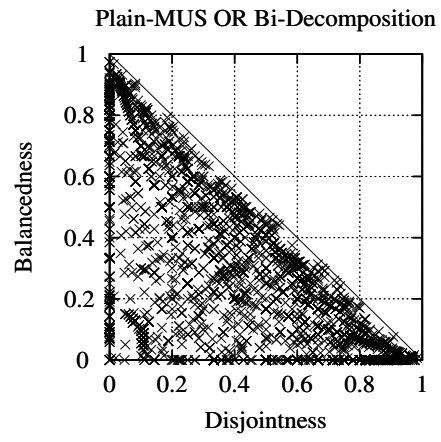

Plain-MUS AND Bi-Decomposition

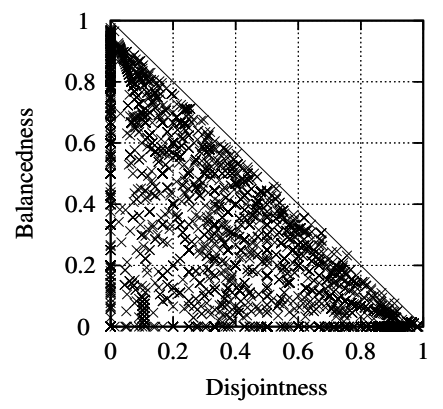

Plain-MUS XOR Bi-Decomposition

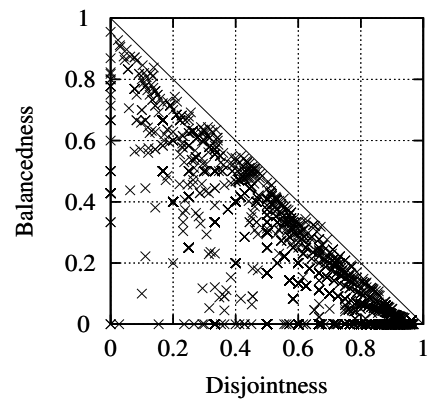

Group-MUS OR Bi-Decomposition

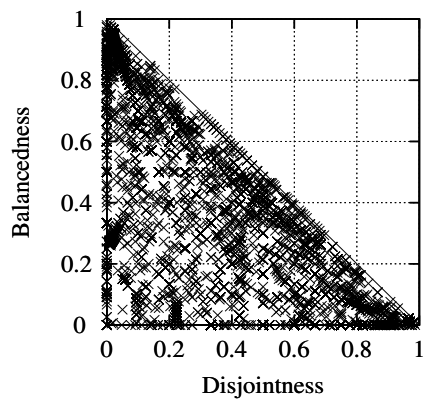

Group-MUS AND Bi-Decomposition

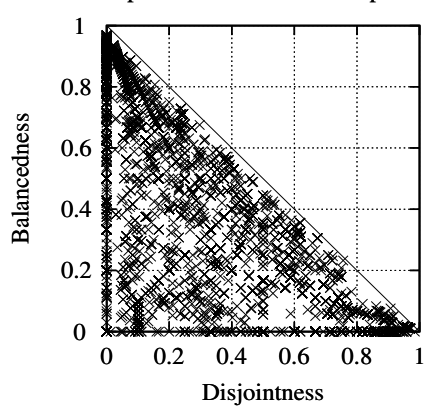

Group-MUS XOR Bi-Decomposition

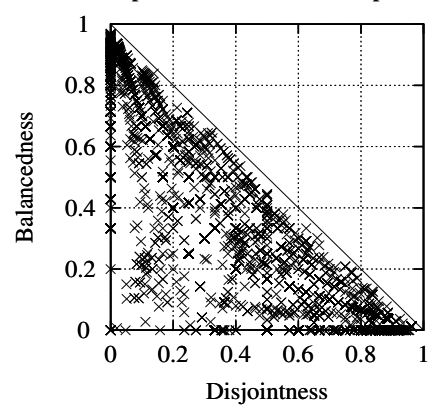

Fig. 9. Quality Metrics for Models 
outperform the original LJH approach [11, achieving similar decomposability. More importantly, the use of group-oriented MUS extraction yields between one and two orders of magnitude speedup for most benchmarks.

Table 2 shows the CPU times and the number of decomposable functions for AND and XOR bi-decompositions for the approaches based on plain and grouporiented MUS extraction. Figure 7 and 8 show the scatter plots comparing the CPU times (in seconds) for each pair of tools for OR, AND and XOR bidecomposition on the ISCAS85, ISCAS89, ITC99 and LGSYNTH benchmark circuits. Each point in each plot represents the CPU time for decomposing a circuit. A more detailed analysis of Figure 7 indicates that the number of aborted circuits for LJH, plain-MUS and group-MUS models are, respectively, 8, 0 and 0 , out of 109 circuits. As can be concluded, both the improved plain and the group-oriented MUS approaches achieve significant performance improvements over the LJH approach, often between one and two orders of magnitude. Moreover, between the two approaches proposed in this paper, the group-oriented MUS approach clearly outperforms the improved plain MUS approach.

\subsection{Quality of Variable Partitions}

The quality of variable partitions mainly determines the quality of bi-decomposition [11. Similar to [11, 13, the quality of a variable partition is measured by two metrics: disjointness and balancedness.

Following [11, disjointness is the preferred metric for measuring the quality of decomposition since a better disjointness corresponds to a smaller number of shared input variables of the resulting decomposed circuit hence potentially yields an optimally decomposed circuit during logic synthesis 11. Similar to 11, $\boldsymbol{S T E P}$ was configured to prefer disjointness over balancedness.

Figure 9 presents the results of quality metrics for LJH OR, Plain-MUS OR/ AND/XOR and Group-MUS OR/AND/XOR models. For XOR bi-decompositions, it has been empirically shown that the LJH approach is unable to achieve good quality decompositions in circuits with regular structures [11. In contrast, the approaches proposed in this paper achieve better disjointness than 11 .

Table 3 compares the quality metrics for different approaches, where only the functions that can be decomposed by the both two competitors are computed. The inferior balancedness of new models compared to LJH model results in part from the mutual exclusion nature between low disjointness and low balancedness

Table 3. Quality metrics comparison between models

\begin{tabular}{|c|c|c|c|c|c|c|c|c|c|c|c|c|c|c|c|}
\hline Metric & OR I & JH vs. & PMUS & OR L & JH vs. & GMUS & OR PI & KUS vs. & GMUS & AND P & MUS v & GMUS & XOR P & MUS vs. & GMUS \\
\hline \multirow[t]{2}{*}{ Disjointness } & \begin{tabular}{||c} 
LJH \\
better
\end{tabular} & Equal & $\begin{array}{l}\text { PMUS } \\
\text { better }\end{array}$ & \begin{tabular}{|c} 
LJH \\
better
\end{tabular} & Equal & $\begin{array}{l}\text { GMUS } \\
\text { better }\end{array}$ & \begin{tabular}{|l} 
PMUS \\
better
\end{tabular} & Equal & $\begin{array}{l}\text { GMUS } \\
\text { better }\end{array}$ & \begin{tabular}{|l} 
PMUS \\
better
\end{tabular} & Equal & $\begin{array}{l}\text { GMUS } \\
\text { better }\end{array}$ & $\begin{array}{l}\text { PMUS } \\
\text { better }\end{array}$ & Equal & $\begin{array}{l}\text { GMUS } \\
\text { better }\end{array}$ \\
\hline & $\mid 24.77 \%$ & $6.40 \%$ & $68.83 \%$ & $36.41 \%$ & $6.40 \%$ & $57.19 \%$ & $\mid 28.88 \%$ & $7.31 \%$ & $63.81 \%$ & $2.56 \%$ & $66.70 \%$ & $30.74 \%$ & $0.70 \%$ & $46.92 \%$ & $52.38 \%$ \\
\hline \multirow[t]{2}{*}{ Balancedness } & \begin{tabular}{||c} 
LJH \\
better
\end{tabular} & Equal & $\begin{array}{l}\text { PMUS } \\
\text { better }\end{array}$ & $\begin{array}{c}\text { LJH } \\
\text { better }\end{array}$ & Equal & $\begin{array}{l}\text { GMUS } \\
\text { better }\end{array}$ & \begin{tabular}{|l} 
PMUS \\
better
\end{tabular} & Equal & $\begin{array}{l}\text { GMUS } \\
\text { better }\end{array}$ & $\begin{array}{l}\text { PMUS } \\
\text { better }\end{array}$ & Equal & $\begin{array}{l}\text { GMUS } \\
\text { better }\end{array}$ & $\begin{array}{l}\text { PMUS } \\
\text { better }\end{array}$ & Equal & $\begin{array}{l}\text { GMUS } \\
\text { better }\end{array}$ \\
\hline & $\mid 35.08 \%$ & $48.57 \%$ & $16.35 \%$ & $47.71 \%$ & $45.66 \%$ & $6.63 \%$ & $\mid 28.32 \%$ & $63.51 \%$ & $8.17 \%$ & $\mid 26.02 \%$ & $61.22 \%$ & $12.76 \%$ & $43.95 \%$ & $47.54 \%$ & $8.51 \%$ \\
\hline
\end{tabular}


for some circuits. As can be observed, the techniques proposed in this paper achieve significantly better disjointness than the LJH approach.

\section{Conclusion}

This paper develops new algorithms for Boolean function bi-decomposition with SAT algorithms. The relative inefficiency of the existing SAT-based models [1] prevent their use on very large industrial circuits. This paper proposes new solutions based on group-oriented MUSes, which have found recent application in hardware design and verification [19 22]. The first improvement builds on the existing SAT-based approach [11, by adding heuristics for improving the quality of partitions and by using more effective MUS extraction algorithms [27. The second improvement consists in formalizing the function bi-decomposition problem in terms of group-oriented MUS extraction [23. Experimental results obtained on representative circuits, demonstrate that the new MUS-based techniques provide significant performance improvements when compared to the earlier work [1], often by more than one order of magnitude. Moreover, the new approaches yield improved quality of results.

Future work will address the integration of $\boldsymbol{S T E P}$ in a logic design flow [39, targeting area, delay and power reduction. Other research directions involve: (i) exploiting $\boldsymbol{S T E P}$ for optimizing circuit networks in LUT (Looked-Up Table) mapping [38; (ii) extending the current models to other forms of decomposition, e.g. Ashenhurst Decomposition [1,13]; (iii) extending the current models for decomposing properties in functional test generation 40; (iv) exploiting SAT-based ATPG (Automatic Test Pattern Generation) [41 and Minimally Unsatisfiable Circuits 42 for identifying and removing redundancy in decomposition; and (v) exploring the optimum variable partition [12, 39, 43, 44] of function decomposition.

Acknowledgment. The authors would like to thank Prof. Jie-Hong Roland Jiang for his helpful comments and for kindly providing the SAT-based Boolean Function bi-decomposition tool $\boldsymbol{B} \boldsymbol{i}$-dec. The authors would like to thank the anonymous referees for helpful comments. This work is partially supported by SFI PI grant BEACON (09/IN.1/I2618).

\section{References}

1. Ashenhurst, R.: The decomposition of switching functions. In: Proceedings of an International Symposium on the Theory of Switching, pp. 74-116 (1957)

2. Curtis, H.: A new approach to the design of switching circuits. Van Nostrand, Princeton (1962)

3. Lai, Y., Pedram, M., Vrudhula, S.: BDD based decomposition of logic functions with application to FPGA synthesis. In: Design Automation Conference, pp. 642-647 (1993)

4. Luba, T., Selvaraj, H.: A general approach to boolean function decomposition and its application in FPGA-based synthesis. VLSI Design 3(3-4), 289-300 (1995) 
5. Scholl, C.: Functional decomposition with application to FPGA synthesis. Springer, Netherlands (2001)

6. Bochmann, D., Dresig, F., Steinbach, B.: A new decomposition method for multilevel circuit design. In: Proceedings of the European Conference on Design Automation, pp. 374-377 (1991)

7. Sasao, T., Butler, J.T.: On bi-decomposition of logic functions. In: International Workshop on Logic and Synthesis, pp. 1-6 (1997)

8. Mishchenko, A., Steinbach, B., Perkowski, M.: An algorithm for bi-decomposition of logic functions. In: Design Automation Conference, pp. 103-108 (2001)

9. Cortadella, J.: Timing-driven logic bi-decomposition. IEEE Transactions on Computer-Aided Design 22(6), 675-685 (2003)

10. Steinbach, B., Lang, C.: Exploiting functional properties of boolean functions for optimal multi-level design by bi-decomposition. Artificial Intelligence Review 20(3), 319-360 (2003)

11. Lee, R.R., Jiang, J.H., Hung, W.L.: Bi-decomposing large boolean functions via interpolation and satisfiability solving. In: Design Automation Conference, pp. 636-641 (2008)

12. Choudhury, M., Mohanram, K.: Bi-decomposition of large boolean functions using blocking edge graphs. In: International Conference on Computer-Aided Design, pp. 586-591 (2010)

13. Lin, H.P., Jiang, J.H., Lee, R.R.: To SAT or not to SAT: Ashenhurst decomposition in a large scale. In: International Conference on Computer-Aided Design, pp. 32-37 (2008)

14. Malik, A., Harrison, D., Brayton, R.: Three-level decomposition with application to PLDs. In: IEEE International Conference on Computer Design: VLSI in Computers and Processors, pp. 628-633 (1991)

15. Sasao, T.: A design method for AND-OR-EXOR three-level networks. In: International Workshop on Logic and Synthesis, pp. 8:11-8:20 (1995)

16. Stanion, T., Sechen, C.: Quasi-algebraic decomposition of switching functions. In: Proceedings of the 16th Conference on Advanced Research in VLSI (ARVLSI), pp. 358-367 (1995)

17. Chang, S., Marek-Sadowska, M., Hwang, T.: Technology mapping for TLU FPGA's based on decomposition of binary decision diagrams. IEEE Transactions on Computer-Aided Design 15(10), 1226-1236 (1996)

18. Jiang, J.H., Lee, C.C., Mishchenko, A., Huang, C.Y.: To SAT or not to SAT: Scalable exploration of functional dependency. IEEE Transactions on Computers 59(4), 457-467 (2010)

19. Liffiton, M., Sakallah, K.: Algorithms for computing minimal unsatisfiable subsets of constraints. Journal of Automated Reasoning 40(1), 1-33 (2008)

20. Khasidashvili, Z., Kaiss, D., Bustan, D.: A compositional theory for post-reboot observational equivalence checking of hardware. In: Formal Methods in ComputerAided Design, pp. 136-143 (2009)

21. Nadel, A.: Boosting minimal unsatisfiable core extraction. In: Formal Methods in Computer-Aided Design (2010)

22. Andraus, Z.S., Liffiton, M.H., Sakallah, K.A.: Refinement strategies for verification methods based on datapath abstraction. In: Asia and South Pacific Design Automation Conference, pp. 19-24 (2006)

23. International SAT Competitions (2002-2011), http://www . satcompetition.org/

24. Cook, S.A.: The complexity of theorem-proving procedures. In: STOC 1971: Proceedings of the Third Annual ACM Symposium on Theory of Computing, pp. 151-158 (1971) 
25. Buning, H.K., Lettman, T.: Propositional Logic: Deduction and Algorithms. Cambridge University Press (1999)

26. Marques-Silva, J.: Minimal unsatisfiability: Models, algorithms and applications. In: International Symposium on Multi-Valued Logic, pp. 9-14 (May 2010)

27. Marques-Silva, J., Lynce, I.: On Improving MUS Extraction Algorithms. In: Sakallah, K.A., Simon, L. (eds.) SAT 2011. LNCS, vol. 6695, pp. 159-173. Springer, Heidelberg (2011)

28. Marques-Silva, J., Sakallah, K.: GRASP-a new search algorithm for satisfiability. In: International Conference on Computer-Aided Design, pp. 220-227 (November 1996)

29. Moskewicz, M., Madigan, C., Zhao, Y., Zhang, L., Malik, S.: Chaff: engineering an efficient SAT solver. In: Design Automation Conference, pp. 530-535 (2001)

30. Eén, N., Sörensson, N.: An Extensible SAT-solver. In: Giunchiglia, E., Tacchella, A. (eds.) SAT 2003. LNCS, vol. 2919, pp. 502-518. Springer, Heidelberg (2004)

31. Biere, A.: PicoSAT essentials. Journal on Satisfiability, Boolean Modeling and Computation (4), 75-97 (2008)

32. Zhang, L., Malik, S.: Validating SAT solvers using an independent resolutionbased checker: Practical implementations and other applications. In: Design, Automation and Test in Europe Conference, pp. 10880-10885 (March 2003)

33. McMillan, K.L.: Interpolation and SAT-Based Model Checking. In: Hunt Jr., W.A., Somenzi, F. (eds.) CAV 2003. LNCS, vol. 2725, pp. 1-13. Springer, Heidelberg (2003)

34. Craig, W.: Linear reasoning. A new form of the herbrand-gentzen theorem. Journal of Symbolic Logic 22(3), 250-268 (1957)

35. Pudlak, P.: Lower bounds for resolution and cutting plane proofs and monotone computations. The Journal of Symbolic Logic 62(3), 981-998 (1997)

36. Cheng, L., Chen, D., Wong, M.: DDBDD: Delay-Driven BDD Synthesis for FPGAs. IEEE Transactions on Computer-Aided Design 27(7), 1203-1213 (2008)

37. Kravets, V., Mishchenko, A.: Sequential logic synthesis using symbolic bidecomposition. In: Design, Automation and Test in Europe Conference, pp. 14581463 (2009)

38. Berkeley Logic Synthesis and Verification Group. ABC: A System for Sequential Synthesis and Verification, Release 70930, http://www.eecs.berkeley.edu/ alanmi/abc/

39. Chen, H., Marques-Silva, J.: New and Improved Models for SAT-Based BiDecomposition. In: Great Lakes Symposium on VLSI (2012)

40. Chen, M., Mishra, P.: Decision ordering based property decomposition for functional test generation. In: Design Automation and Test in Europe, pp. 167-172 (2011)

41. Chen, H., Marques-Silva, J.: TG-PRO: A SAT-based ATPG System, System Description. Journal on Satisfiability, Boolean Modeling and Computation 8, 83-88 (2012)

42. Belov, A., Marques-Silva, J.: Minimally Unsatisfiable Boolean Circuits. In: Sakallah, K.A., Simon, L. (eds.) SAT 2011. LNCS, vol. 6695, pp. 145-158. Springer, Heidelberg (2011)

43. Chen, H., Janota, M., Marques-Silva, J.: QBF-Based Boolean Function BiDecomposition, Computing Research Repository (CoRR), abs/1112.2313 (December 2011)

44. Chen, H., Janota, M., Marques-Silva, J.: QBF-Based Boolean Function BiDecomposition. In: Design Automation and Test in Europe (2012) 